\title{
Radiology
}

\section{The Phantom for Studying Foreign Bodies' Echo-Signs}

\author{
Ekaterina G. Privalova, $\mathrm{PhD}^{1}$; Yana A. Shumina ${ }^{1}$; \\ Aleksandr Yu. Vasilyev, $\mathrm{PhD}, \mathrm{ScD}^{1,2^{*}}$; Igor N. Bondarenko, $\mathrm{PhD}^{1}$ \\ ${ }^{1}$ Central Research Institute of Radiation Diagnostics, Moscow, Russia \\ ${ }^{2}$ Moscow State University of Medicine and Dentistry named after A. I. Evdokimov, Moscow, Russia
}

\begin{abstract}
Background: The frequency of foreign bodies (FBs) is about 4\% among all diseases in the maxillofacial region and more than a third of them are missed during initial examinations. Several authors have found that multi-position ultrasound examination of the soft tissues of the maxillofacial region has a high diagnostic significance. The aim of our study was to create a useful simulation model of soft tissues from durable material to study the echo-signs of FBs and to confirm ultrasonography as a method of choice for suspicion of the presence of FBs in the soft tissues of the maxillofacial region.

Methods and Results: We created a utility model. Two parts of a phantom were made of silicone-containing material with organic and non-organic FBs located in two parallel rows at a depth of $5 \mathrm{~mm}$ and $20 \mathrm{~mm}$. The echo-signs of objects of organic and non-organic origin were determined. Ultrasonography allowed visualization of all FBs located at a depth of $5 \mathrm{~mm}$. None of the organic FBs placed into the phantom were visualized by computed tomography. Compared with computed tomography, ultrasonography was more informative and preferable in the diagnosis of FBs of soft tissues.

Conclusion: The created model is a highly sensitive and accurate tool for detecting even the radiolucent FBs of different origin. (International Journal of Biomedicine. 2020;10(2):124-128.)
\end{abstract}

Key Words: ultrasonography $\bullet$ soft tissues $\bullet$ model $\bullet$ foreign bodies

\section{Abbreviations}

3DR, 3D reconstruction; CT, computed tomography; MRI, magnetic resonance imaging; MPR, multiplanar reconstruction; MIP, maximum intensity projection; US, ultrasonography

\section{Introduction}

Foreign bodies (FBs) in the soft tissues of the maxillofacial region are a common pathology with which patients turn to dentists, surgeons and radiologists. The frequency of FBs is about $4 \%$ among all diseases in the maxillofacial region. ${ }^{(1)}$ About $30 \%$ of all FBs remain undiagnosed during the initial examinations. ${ }^{(2)}$ They are detected accidentally by ultrasonography (US), or purposefully after trauma or after the onset of symptoms. In some cases, patients are referred to ultrasound for follow-up after computed tomography (CT)

"Corresponding author: Prof. Alexander Yu. Vasilyev, MD, PhD, ScD. Department of Radiology, Moscow State University of Medicine and Dentistry named after A. I. Evdokimov, Moscow, Russia.E-mail:auv62@mail.ru or magnetic resonance imaging (MRI) because of its high resolution and sensitivity of $95 \%$ for detection of $\mathrm{FBs}^{(3)}$

FBs could be divided into two groups: of organic and non-organic origin. It is highly important to determine the nature of FBs because their nature affects the strategy of patient management and surgical treatment planning.

There are only a few scientific works on this theme, and there are still no precise echo-signs of FBs of the maxillofacial area, despite the obvious urgency, a high risk of complications and significant interest in patients with the presence of FBs in the soft tissues.

The aim of our study was to create a useful simulation model of soft tissues from durable material to study the echosigns of FBs and to confirm US as a method of choice for suspicion of the presence of FBs in the soft tissues of the maxillofacial region. 


\section{Materials and Methods}

Phantom features

We created a utility model. Two parts of a phantom were made of silicone-containing material with organic and nonorganic FBs located in two parallel rows at a depth of $5 \mathrm{~mm}$ and $20 \mathrm{~mm}$. The phantom for assessing the FB echo-signs of organic and non-organic origin consists of two bodies and 20 small parts. The bodies of the phantom are made of siliconecontaining material and have the form of a cuboid measuring $210 \times 50 \times 100 \mathrm{~mm}$. Placed inside, FBs are arranged in pairs in two parallel rows at a depth of $5 \mathrm{~mm}$ and $20 \mathrm{~mm}$. The material of which the phantom consists has acoustic properties similar to the soft tissues of a person with a hypersthenic type of build $(\sim 1450 \mathrm{~m} / \mathrm{s})$. The material is durable, as it does not contain gelatin, glycerine or other materials usually used in the creation of ultrasound phantoms.

The first phantom (with non-organic FBs) includes the following parts (Fig.1):

1. Glass splinters $(n=2)$

2. Fragments of plastic $(n=2)$

3. Drain tubes $(n=2)$

4. Metal screws $(\mathrm{n}=2)$

4. Dental filling material $(\mathrm{n}=2)$

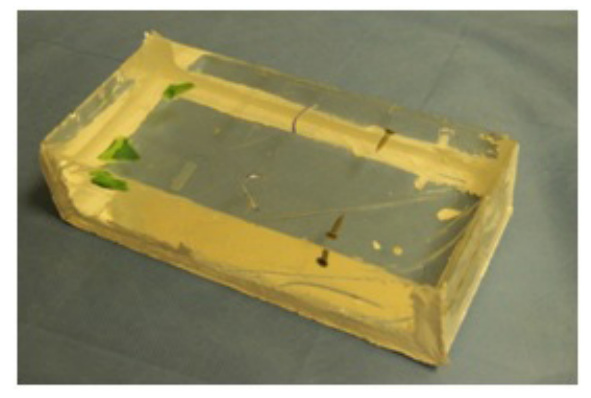

Fig. 1. The phantom with non-organic FBs.

The second phantom (with organic FBs) includes the following parts (Fig.2):

1. Husks of a sunflower seed $(\mathrm{n}=2)$

2. Rose thorns $(\mathrm{n}=2)$

3. Blades of grass $(n=2)$

4. Pieces of a wooden toothpick $(\mathrm{n}=2)$

5. Helminths $(n=2)$

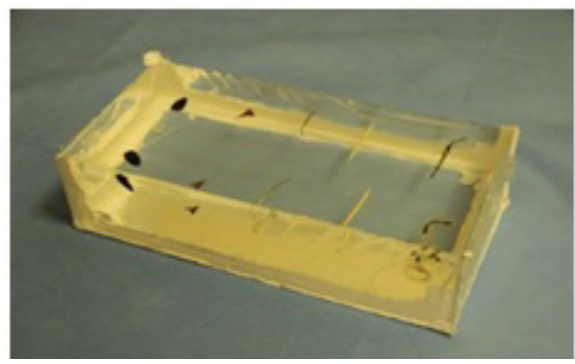

Fig. 2. The phantom with organic FBs.

The variety of objects allows establishing the echo-signs of each material, both organic and non-organic, including their artifacts.
Methods

Both phantoms underwent US and CT. US was performed on an IU-22 device (Philips, the Netherlands) using a pencil transducer, with a frequency of $7-15 \mathrm{MHz}$ and a linear transducer with a frequency of 5-17 MHz in B-mode (Fig.3).

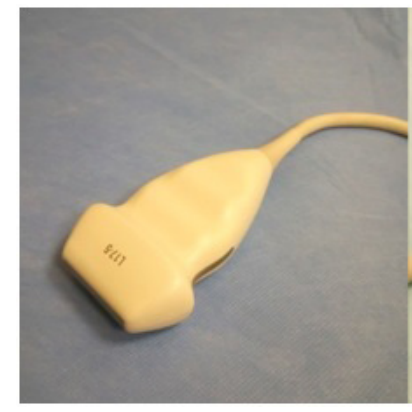

(a)

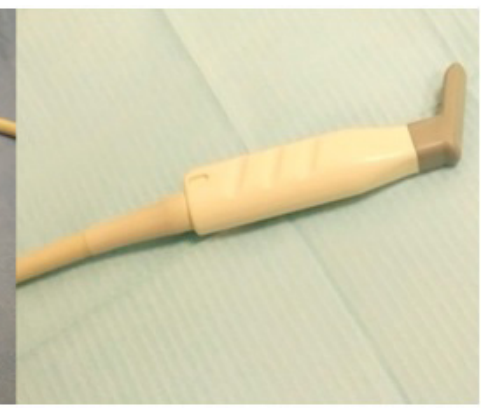

(b)
Fig. 3. Linear (a) and pencil (b) transducers.

A special gel pillow was used for ultrasound examinations to improve the visualization (Fig.4), then a CT was performed on both phantoms by a 64-slice tomograph with the evaluation of the obtained images in the axial plane, using MPR, MIP, and 3DR (Fig.5).

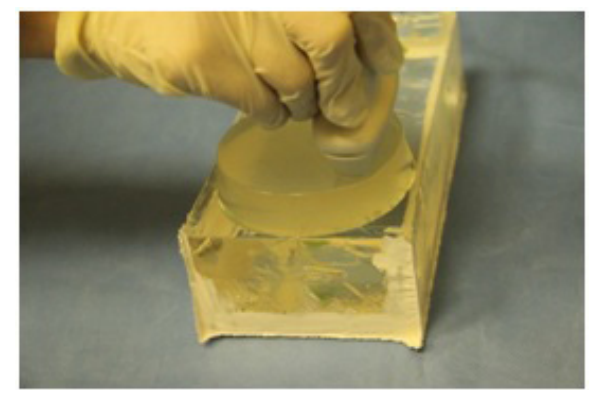

Fig.4. The gel pillow.

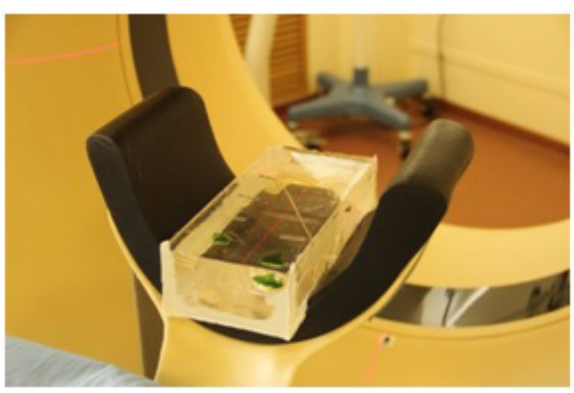

Fig. 5. CT of the model.

\section{Results and Discussion}

\section{Ultrasonography}

As a result of the research, the following ultrasound picture of the phantom was visualized: three layers separated by two layers. The upper layer (gel pillow) had increased echogenicity, a quite inhomogeneous echostructure because of tiny inclusions. The first interlayer, between the gel pillow and the top layer of the model, was visualized like a hyperechoic linear structure with a slight reverberation artifact. The second 
layer (the upper layer of the model, located at a depth of 0-20 $\mathrm{mm}$ ) was visualized as an anechoic, moderately homogeneous space with single inclusions (artifacts). The second interlayer (between two layers of the model) was visualized as a hyperechoic linear structure. The third layer (the bottom layer of the model, located at a depth of 20-50 mm) was visualized as an anechoic layer (Fig.6).

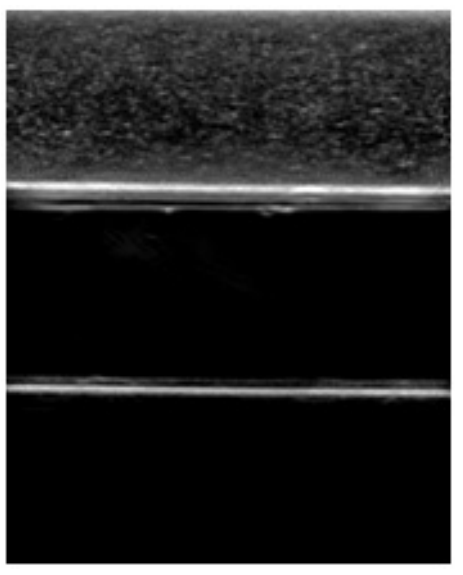

Fig. 6. US picture of the model (part without FBS)

1 - gel pillow, 2 -the first interlayer, 3 - the upper layer of the model, 4 - the second interlayer, 5 - the bottom layer of the model.

All FBs located at a depth of $5 \mathrm{~mm}$ were clearly visualized. But due to technical aspects (increased material density and physical properties of the interlayer), objects located at a depth of $20 \mathrm{~mm}$ were not convincingly visualized. Non-organic FBs were glass splinters $(n=2)$, fragments of plastic $(n=2)$, drain tubes $(n=2)$, metal screws $(n=2)$, and dental filling material $(\mathrm{n}=2)$.

The glass splinter was visualized as an isoechoic structure with hyperechoic, even sometimes fuzzy contours, a reverberation artifact and acoustic amplification (Fig.7). The fragment of plastic was visualized as a linear anechoic structure with clear and even hyperechoic contours (Fig.8). The drainage tube was visualized as a curly linear anechoic structure with clear and quite uneven hyperechoic contours (Fig.9). The metal dental screw was visualized as a structure with alternating bands of high and low echogenicity, with clear uneven contours and artifacts such as posterior shadow and reverberation (Fig.10). The dental filling material was visualized as a hyperechoic structure with unclear and quite uneven contours and a reverberation artifact (Fig.11).

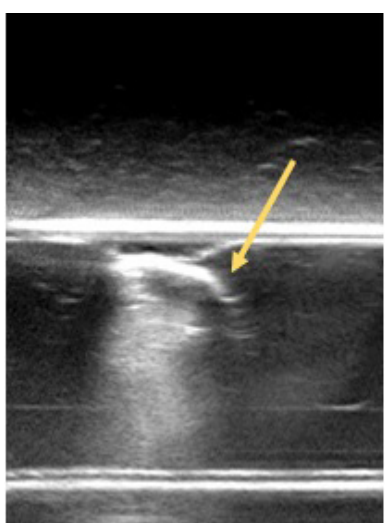

Fig. 7. US of the model. B-mode. The glass splinter.

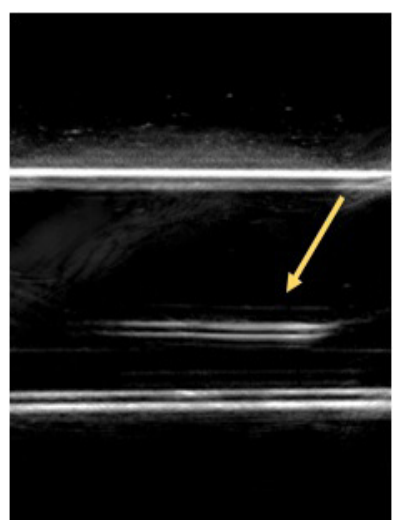

Fig. 8. US of the model. B-mode The fragment of plastic.

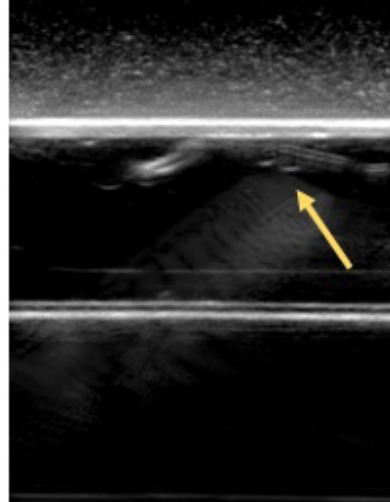

Fig. 9. US of the model. $B$-mode. The drainage tube.

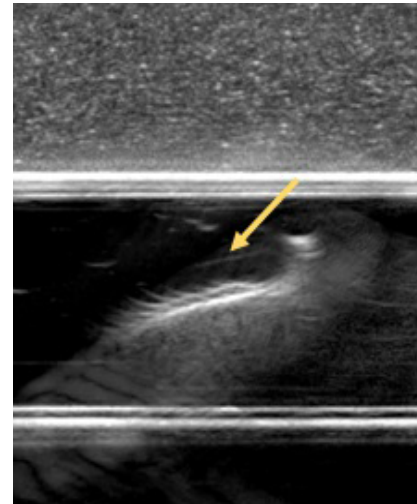

Fig. 10. US of the model. B-mode. The dental screw.

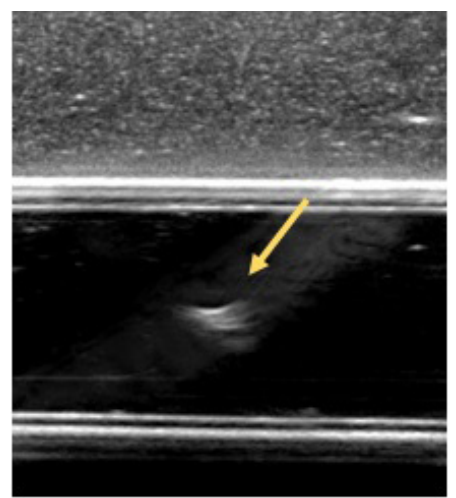

Fig. 11. US of the model. B-mode. The dental filling material.

Organic FB were husks of a sunflower seed $(n=2)$, rose thorns $(n=2)$, blades of grass $(n=2)$, pieces of a wooden toothpick $(n=2)$, and helminths $(n=2)$.

The husk of a sunflower seed was visualized as a linear hyperechoic structure with even and sufficiently clear contours. It was also possible to trace the curved shape of the husk (Fig.12). The rose thorn was visualized as a structure that looked like an elongated triangle, with increased echogenicity and clear, even contours and homogeneous echostructure (Fig.13). The blade of grass was visualized as a curly structure with increased echogenicity with even and somewhat unclear contours (Fig.14). The piece of a wooden toothpick was visualized as a linear hyperechoic structure with clear and even contours (Fig.15).

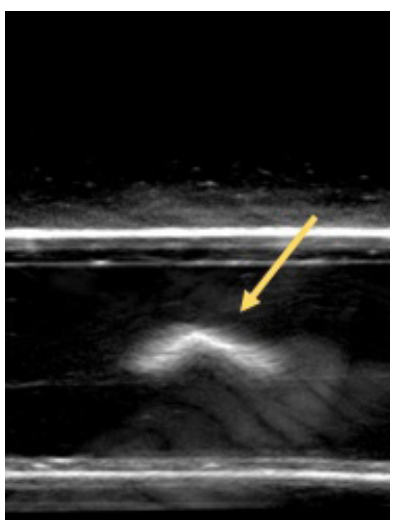

Fig. 12. US of the model. B-mode. The husk of a sunflower seed.

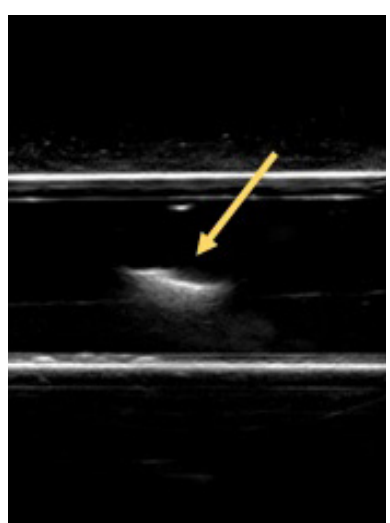

Fig. 13. US of the model. $B$-mode. The rose thorn. 

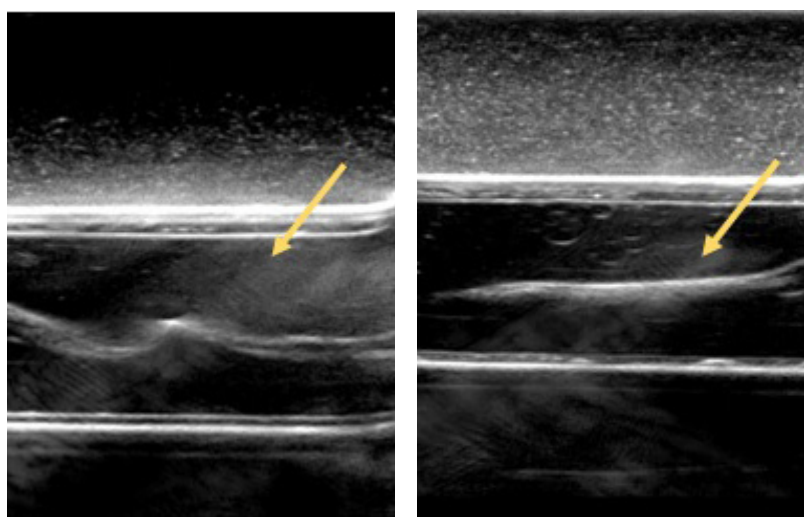

Fig.14. US of the model. B-mode. Fig.15. US of the model. B-mode The blade of grass. The piece of a wooden toothpick.

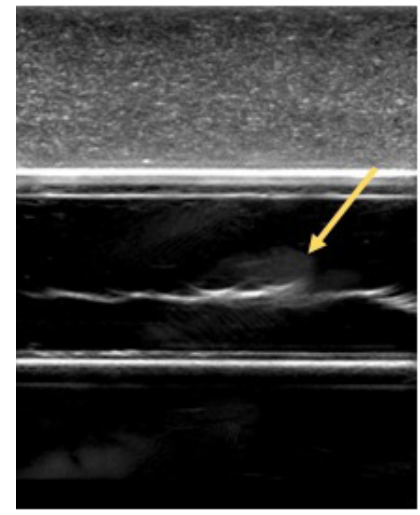

Fig. 16. US of the model. B-mode. The helminth.

The helminth (dead) was visualized as a curved structure with increased echogenicity contours and hypoechoic content. When the halminth was strongly decomposed, it was visualized as an anechoic tubular cavity with contours of somewhat increased echogenicity and with heterogeneous hyperechoic content along the lower edge - it is detritus resulting from the decomposition of the helminth (Fig.16). If the helminth were alive, its movement and peristalsis would be determined in Doppler modes.

US allowed visualization of all FBs located at a depth of 5 $\mathrm{mm}$. The visualization of structures located at a depth of $20 \mathrm{~mm}$ was difficult due to the technical aspects (increased material density and physical properties of the interlayer). In addition, US allowed estimation of the echo-signs of all FBs, with the exact characteristics of the echo-signs, including artifacts.

\section{Computed tomography}

None of the organic FBs placed into the phantom were visualized by CT (Fig.17).

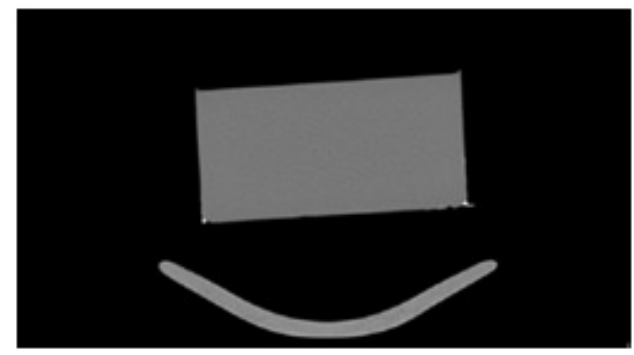

Fig.17. CT of the model with organic FBs.
However, glass splinters, metallic dental screws and a filling material were visualized by the $\mathrm{CT}$ of the phantom with non-organic FBs (Fig.18-20).

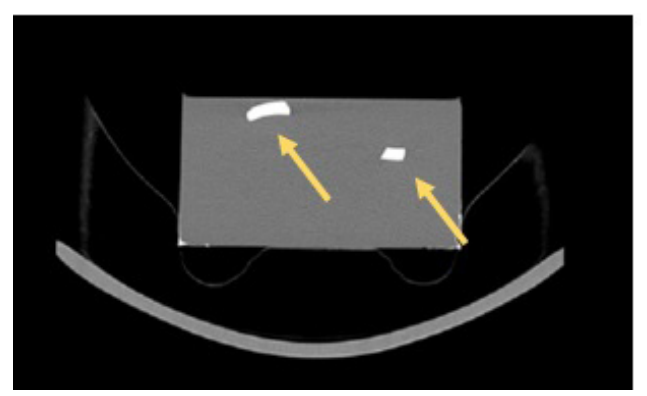

Fig. 18. CT of the model. Glass splinters.

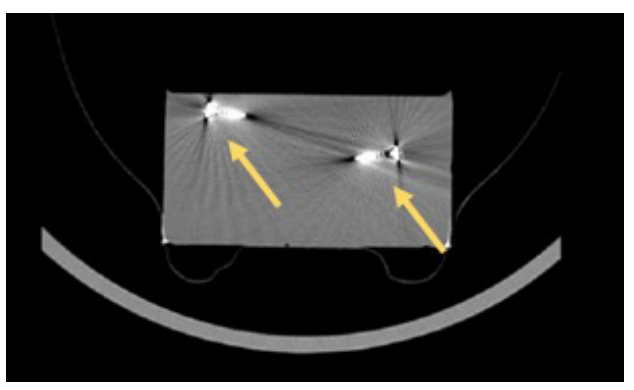

Fig. 19. CT of the model. Metallic dental screws.

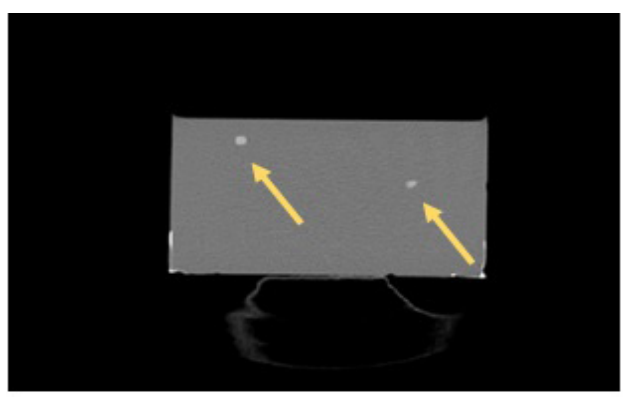

Fig. 20. CT of the model. The dental filling material.

A 3DR was presented for clarity (Fig.21). There were also zones with air in both models, which got there during manufacturing (Fig.22). High density materials, such as metal dental screws, filling material and glass splinters, were clearly detected. Drain tubes and fragments of plastic were not visualized because they are radiolucent.

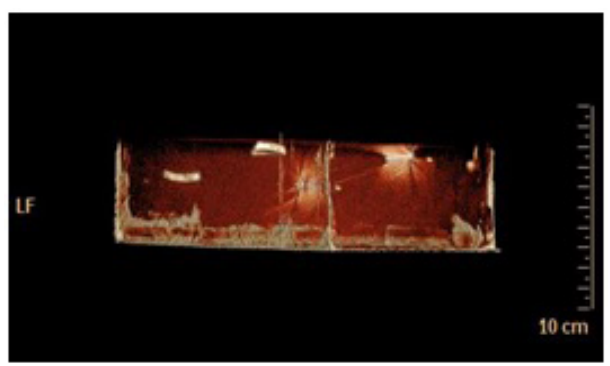

Fig. 21. 3DR. Glass splinters, dental screws and filling material were visualized. 


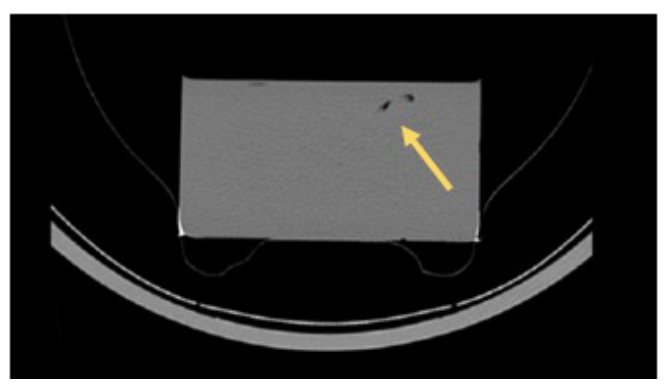

Fig. 22. CT of the model. Air.

In conclusion, as a result of the experiment, a utility model was created for the study of ultrasonic signs of FBs of various origin, which can be a useful tool for this type of study. The echo-signs of objects of organic and non-organic origin were determined. Compared with CT, US was more informative and preferable in the diagnosis of FBs of soft tissues. Thus, the created model is a highly sensitive and accurate tool for detecting even the radiolucent FBs of different origin.

\section{Competing Interests} interests.

The authors declare that they have no competing

\section{References}

1. Valizadeh S, Pouraliakbar H, Kiani L, Safi Y, Alibakhshi L. Evaluation of Visibility of Foreign Bodies in the Maxillofacial Region: Comparison of Computed Tomography, Cone Beam Computed Tomography, Ultrasound and Magnetic Resonance Imaging. Iran J Radiol. 2016;13(4):e37265. doi: 10.5812/ iranjradiol.37265.

2. Mohamed A, Varma B, Valappila NJ, Meena SA. Entrapped foreign body: A diagnostic muddle for the radiologist. Indian $\mathrm{J}$ Dent. 2016 Sep;7(3):158-161.

3. Tantray MD, Rather A, Manaan Q, Andleeb I, Mohammad M, Gull Y. Role of ultrasound in detection of radiolucent foreign bodies in extremities. Strategies Trauma Limb Reconstr. 2018 Aug;13(2):81-85. doi: 10.1007/s11751-0180308-z. doi: 10.1007/s11751-018-0308-z. 